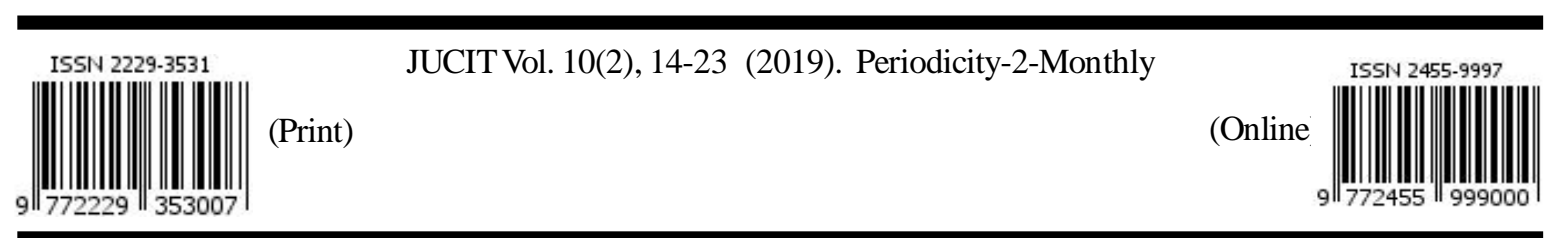

JOURNAL OF ULTRA COMPUTER \& INFORMATION TECHNOLOGY
An International Open Free Access Peer Reviewed Research Journal of Computer
Science Engineering \& Information Technology
website:- www.compitjournal.org
Estd. 2010

\title{
Automated Workflow Execution of Loan Transaction Processing for Distributed Environment
}

\author{
JOHN-OTUMU, A. M ${ }^{1}$, IMHANLAHIMI, R. E ${ }^{2}$ and OSHOIRIBHOR, E. O \\ ${ }^{1}$ Training, Research \& Innovations Unit, Directorate of ICT, Ambrose Alli University, Ekpoma (Nigeria) \\ ${ }^{2,3}$ Department of Computer Science, Ambrose Alli University, Ekpoma (Nigeria) \\ Corresponding author: macgregor.otumu@gmail.com \\ http://dx.doi.org/10.22147/jucit/100202
}

Acceptance Date 15th April, 2019, Online Publication Date 21st April, 2019

\begin{abstract}
This research work addresses the daily challenges encountered by loan applicants requesting for loan facilities from a loan scheme and the unnecessary end-to-end delays in the processing and some time the computational errors associated with the conventional ways of handling these task. An Automated Workflow Execution of loan transaction processing for distributed environment was designed as a result of the shortcomings attributed to the traditional system/environment for loan processing, web development tools such as Hypertext Markup Language (HTML), Hypertext Preprocessor (PHP), JavaScript, and MySQL were used to realize the web interface, and the distributed Automated Workflow Execution Engine for loan transaction processing. We achieved our objective in designing of the distributed software architecture and developing the web based automated workflow execution system to solve local loan transaction problems. A comparative evaluation in terms of features and functionalities was also done between the proposed system and five commercial automated workflow systems. Results revealed that the proposed system has lightweight architecture in terms of design patterns and the development tools used.
\end{abstract}

Key words: Distributed environment, automated workflow, business processes, loan transaction

\section{Introduction}

Workflow is the definition, execution and automation of corporate procedures where jobs, information or documents are approved from one member to another for action, according to a set of routine instructions ${ }^{11}$. A workflow system is used to describe corporate procedure jobs at a conceptual level necessary for understanding, evaluating, and redesigning a business process. Workflows captures information process tasks at a level that describes the process requirements for information system functionality and human skills ${ }^{15}$.

Establishments use workflows to organize

This is an open access article under the CC BY-NC-ND license (https://creativecommons.org/licenses/by-nc-sa/4.0) 

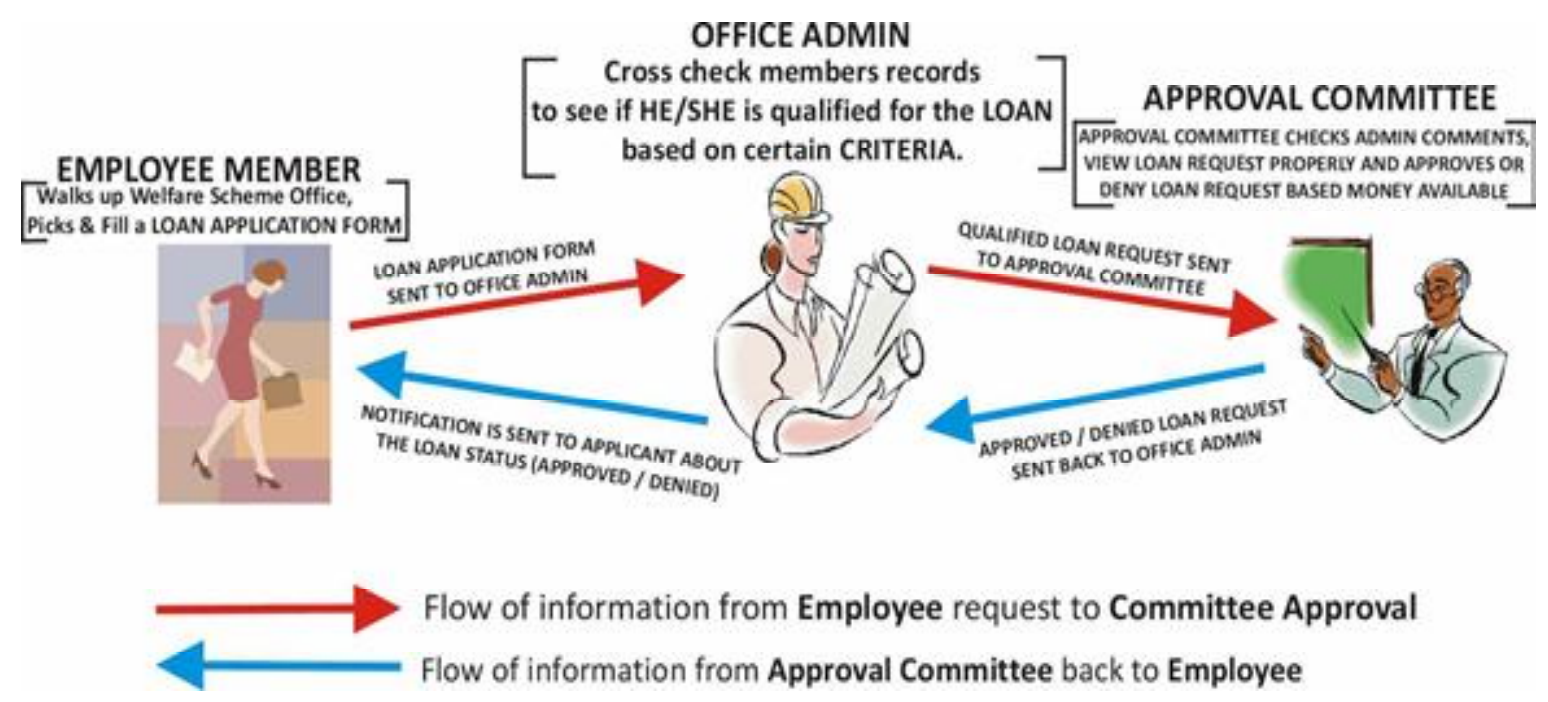

Figure 1: Existing loan scheme manual transaction workflow process

jobs between people and coordinate data between systems, with the ultimate goal of improving organizational efficiency, responsiveness and profitability. Workflows systematize the flow of employee tasks and doings, reducing the time the process took to complete as well as possible mistakes caused by human collaboration.

Workflows make processes more effective, agile, and visible by guaranteeing that every process step is explicitly defined, monitored over time, and optimized for maximum productivity. Workflows empower business users and Information Technology to work together to rapidly modify systems and processes to reflect changes in the business.

Over the years, workflow technology has been applied to mobile computing, systems management, multi databases, Internet, object technology, operating systems, and transaction management $^{15}$.

Many workflow management systems meant for commercial and research purpose support distributed workflow execution and some of them are temporarily adapted. For example workflow system like ADEPT ${ }^{24}$, Exotica ${ }^{19}$, ORBWork ${ }^{7}$, Rainman ${ }^{22}$ and Newcastle-Nortel ${ }^{27}$ are some of the distributed workflow management systems available from literature reviewed. Reichert et al. $^{25}$; Ranno and Shrivastava ${ }^{23}$ exhaustively discussed and described qualities of a good distributed workflow management systems.

The research paper enables the sharing, computation and archival of information as loan transaction processing is transferred from one desk to another until the process is fully executed. These tasks are executed when the relevant office receives a request containing the relevant information to be treated in a paper trail during office hours only.

It is observed that the conventional procedures for requesting or applying for loan facility to the process of granting the loan takes a lot of time due to the very rigid and sluggish procedures associated with the traditional method.

Figure 1 depicts a diagrammatic manual loan application / approval workflow process of the welfare and loan scheme.

The inability for applicants to apply for loan facilities from the comfort of their homes and also track the status of their application at any point in time and from any part of the country or world is a major objective in which this research work intends to fill by developing an automated workflow system for loan processing in a distributed environment.

\section{Literature Review :}

Kowalski ${ }^{14}$ proposed the Event Calculus as 
a logic-based methodology for specification and execution of workflows in the context of database applications. Also, several researchers like ${ }^{5,16,21}$ have worked on artifact-centric or data-centric workflows.

There are many researchers like $1,2,3,4,10,13,17$ who have explicitly focused on the problem of verifying the correctness of inter-organizational workflows in the domain of petri nets. Aalst ${ }^{1}$ used message sequence charts to model the interaction between the participant workflows using petri nets and the overall workflow is checked for consistency against an interaction structure specified in message sequence charts.

Kindler et al. ${ }^{13}$ followed a similar but more formal and concrete approach, where the interaction of different workflows is specified using a set of scenarios given as sequence diagrams and using criteria of local soundness and composition theorem, guaranteed the global soundness of an interorganizational workflow.

Hofstede et al. ${ }^{10}$ proposed Query Nets based on predicate/transition petri nets to guarantee global termination, without the need for having the global specification. The work on workflow nets use a P2P (Public-To-Private) approach to partition a shared public view of an inter-organizational workflow over its participating entities and projection inheritance is used to generate a private view that is a subclass to the relevant public view, in order to guarantee the deadlock and livelock of freedom.

Modeling global behavior as a set of conversations among participating services has been studied by research scholars such as ${ }^{6,9,26,28,29}$ in the areas of business processes.

According to $\mathrm{Fu}$ et al. ${ }^{9}$, an approach based on guarded automata for the realization and analysis of the conversation protocols, whereas the authors in $^{29}$ used colored petri nets to capture the complex conversations.

Researchers like $e^{8,12,18,20}$ in the web services community have been working on web service composition and decentralized process execution of workflow using BPEL and other related technologies to model the web services. A technique to partition a composite web service using program analysis was studied by ${ }^{20}$ and a similar approach by ${ }^{12}$ explored decomposition of a business process modeled in BPEL, primarily focusing on $\mathrm{P} 2 \mathrm{P}$ interactions. Using a formal approach based on input / output automata representing the services, the authors in ${ }^{18}$ also studied the problem of synthesizing a decentralized choreography strategy that will have optimal overhead of service composition in terms of costs associated with each interaction.

\section{Methodology :}

This section covers the automated workflow architecture for loan transaction processing, and the development tools used in building the proposed workflow system.

Figure 2 depicts the software architectural design of the proposed system. Process here is a program in execution to perform some kind of task, and the process definition of this software is built using a hierarchical decomposition method in order to control the complexity of the design. The processes in this design are also structured to operate on different and several levels. The login page accepts login parameters from users from different sites or location requesting for access to the workflow application through the Internet. The global users interface handler accepts the request and its parameters from the distributed environment for site authentication before sending the request to the next phase which is the central login monitor handler which monitors and performs users authentication by verifying the parameters entered with details it has in the monitor tables before granting the different users access, rights and priviledges. Users can then perform certain actions based on their rights / priviledges also; these actions or events are processed by the event handler which sends and receives actions to and fro the automated workflow system engine (AWfs Engine) after processing. The event handler commits such actions to the database for storage, and also sends events messages to queue of events for onward transmission to the various users through the dispatchers. 


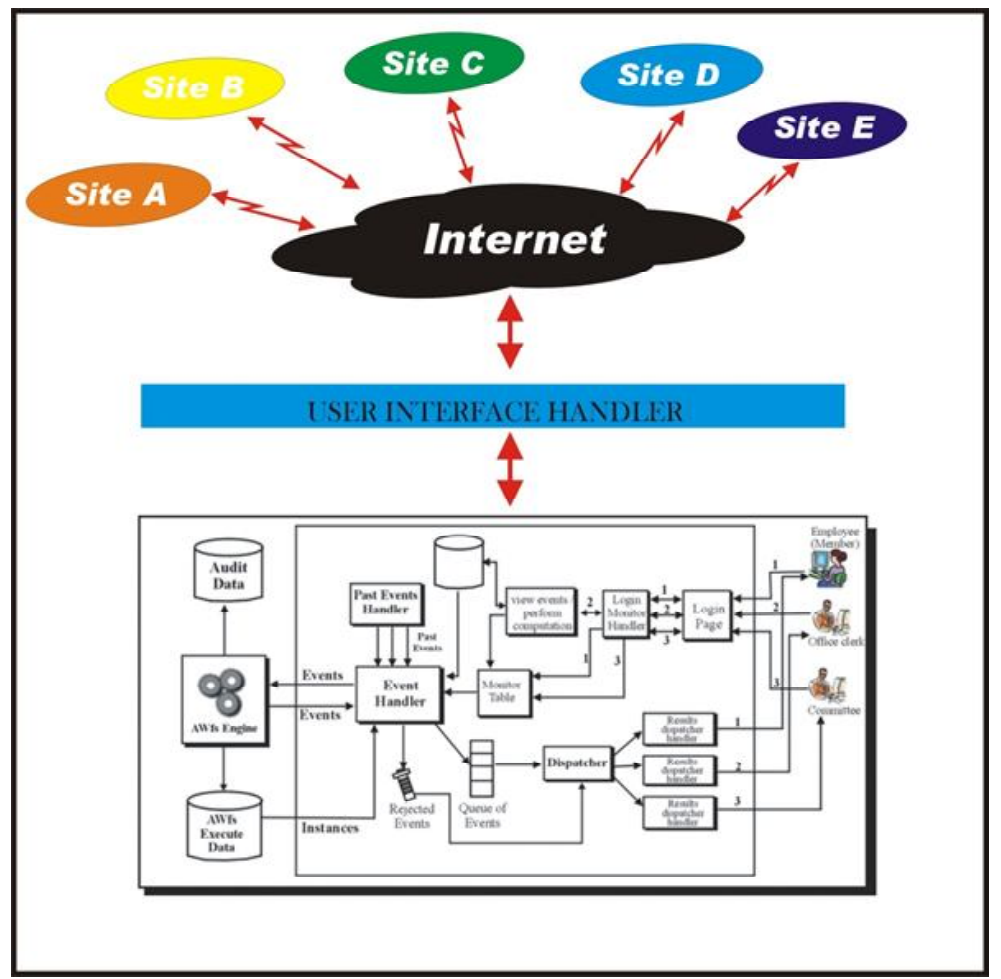

Figure 2: Proposed Automated Workflow Architecture Adapted from (John-Otumu, Okonigene \& Imhanlahimi, 2015)

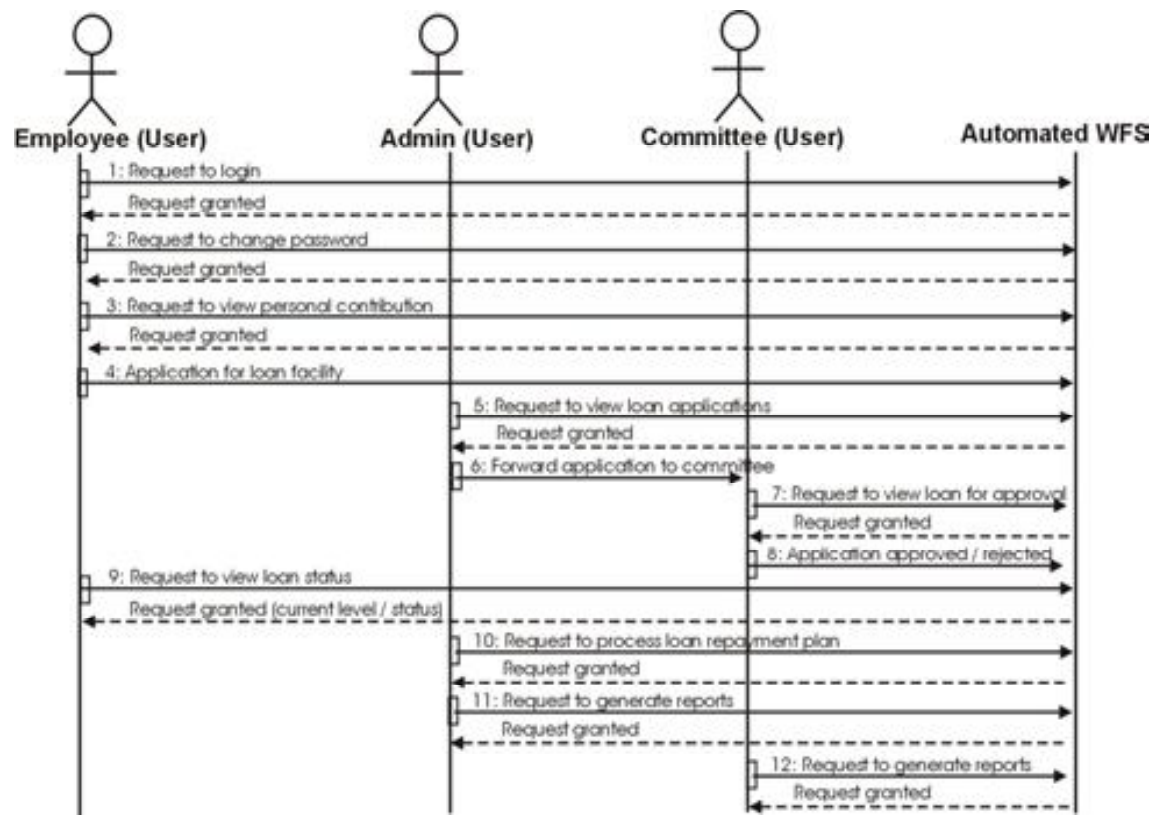

Figure 3: Sequence diagram showing the loan transaction interaction process 
The proposed automated workflow system was built using web development tools like Hypertext Preprocessor (PHP), JavaScript, Hypertext Markup Language and MySQL. These web languages were carefully selected because of their strengths and the purpose the researcher needed to achieve.

\section{Results and Discussion}

This section discusses the results obtained from developing and implementing the automated workflow system based on the designed architecture.

Figure 4 shows the online loan request form. Here, applicants requesting for loan facility can use the tab key to transverse between the various fields displayed in the proper order, both in forward directions using only the tab key and in backward directions combining the shift + tab key.

Figure 5 shows the online membership application form created by the office admin. The office admin can also use the tab key to transverse between the fields displayed in forward directions using only the tab key and in backward directions combining the shift + tab key. The online membership application form can also trap invalid data correctly, especially date, numeric, and alphanumeric formats because the fields were declared with different data types, and validated to accept a specified input field length of data at the database table structure design level.

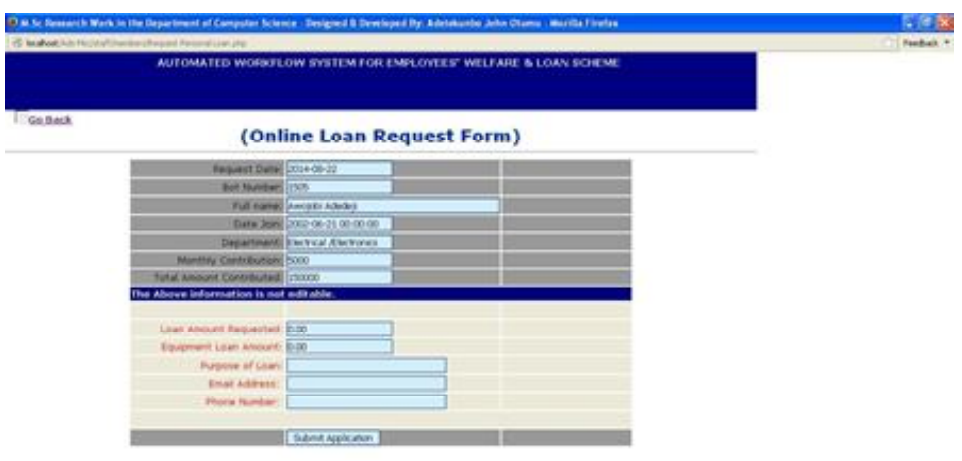

Figure 4: Online Loan Request Form

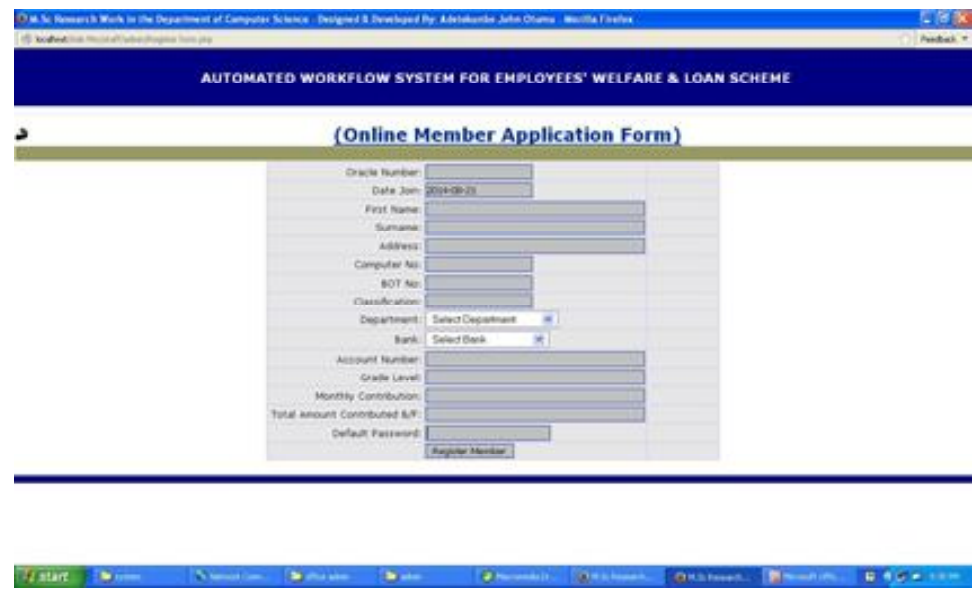

Figure 5: Online Membership Application Form 


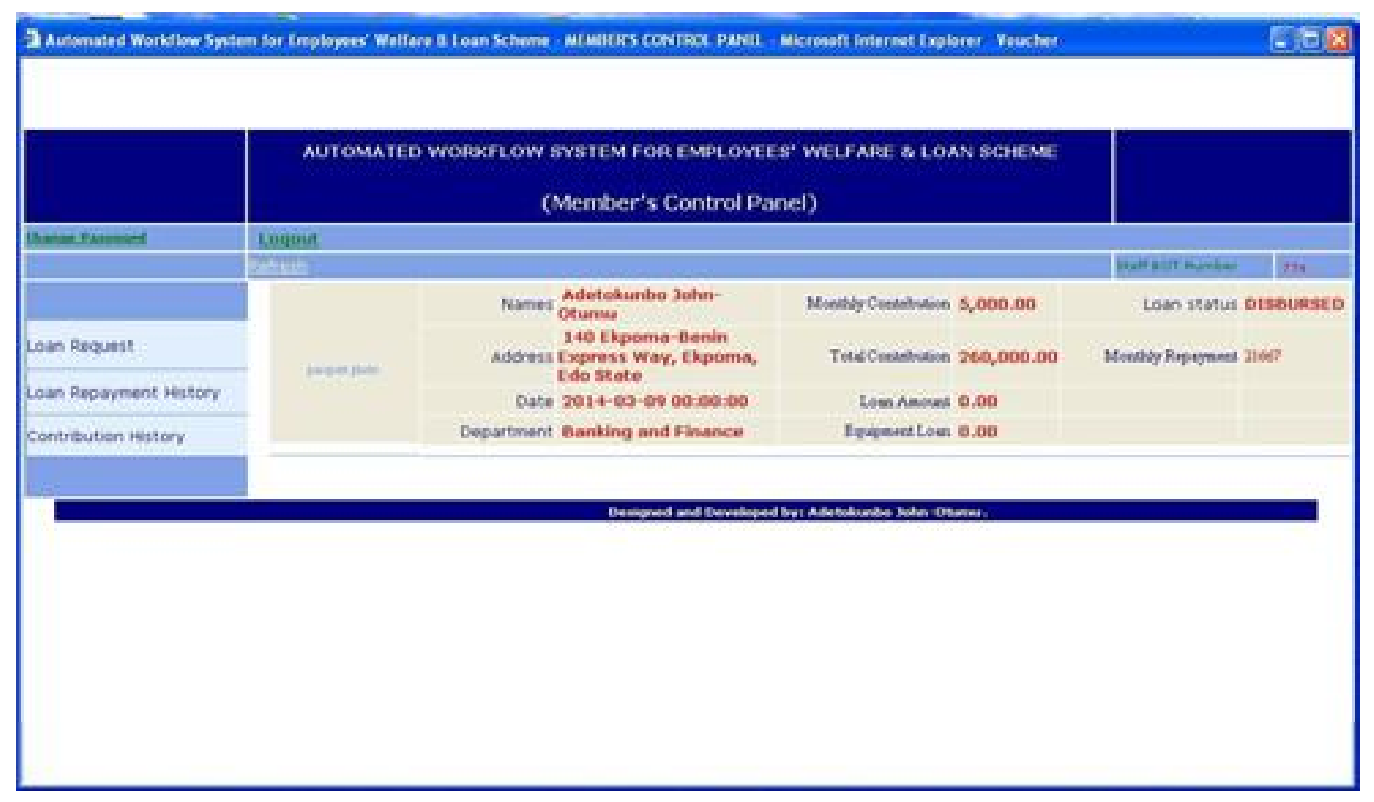

Figure 6: Applicant's control panel
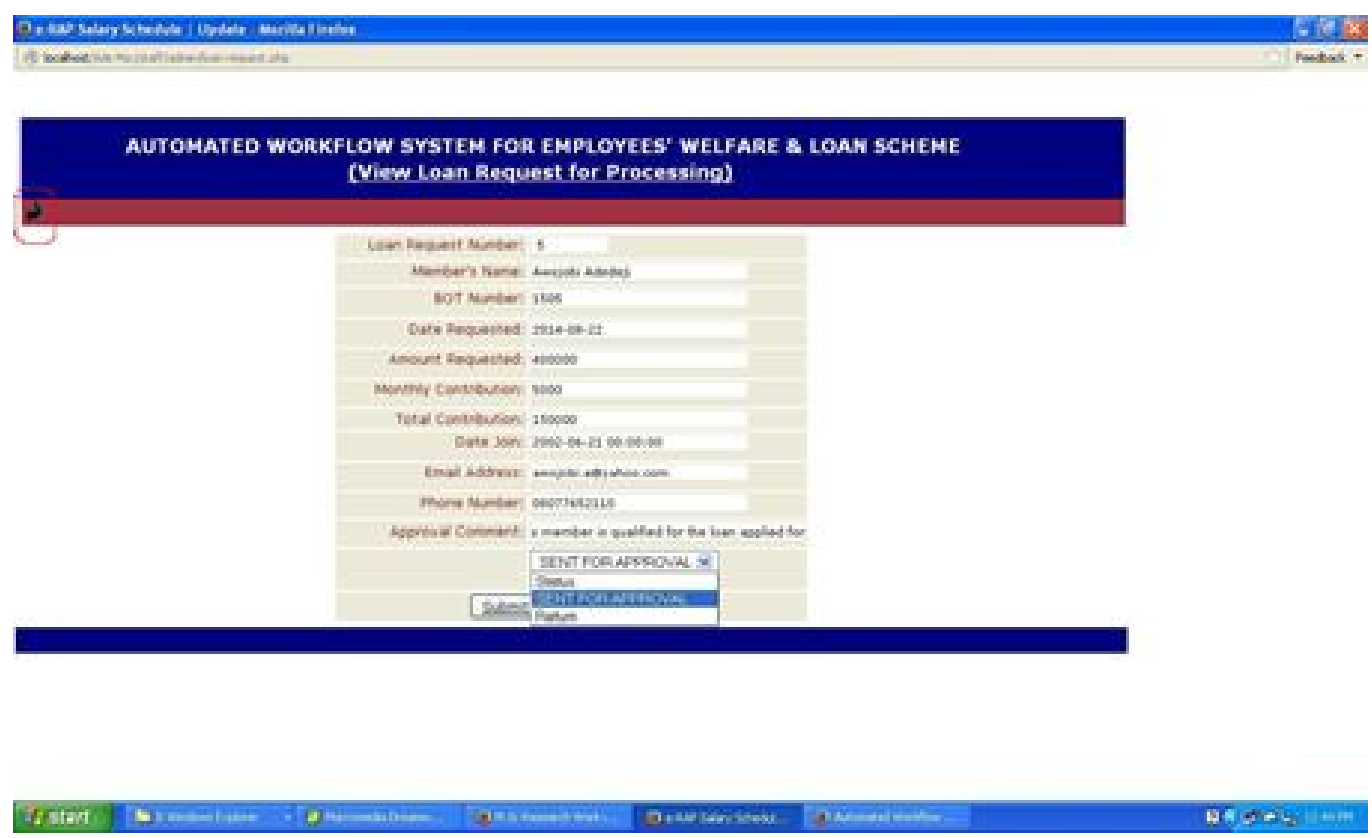

Figure 7: View loan request for processing interface

Figure 6 shows a user (member's) control panel with user's information displayed as a result of the correct authentication process. Here the user can make loan request, view status, repayment and contribution history.

Figure 7 shows the interface for viewing loan request with a hyperlink icon on the left hand side to move back to previous page when clicked upon. 
Table 1: Comparative evaluation between the proposed automated workflow system and five commercial workflow

\begin{tabular}{|c|c|c|c|c|c|c|}
\hline Feature / Product & \begin{tabular}{|c|} 
Our Developed \\
Automated \\
Workflow \\
System
\end{tabular} & InConcert & Staffware & FloWare & Notes & $\begin{array}{c}\text { Action } \\
\text { Workflow }\end{array}$ \\
\hline Classification & Administrative & Production & Administrative & $\begin{array}{c}\text { Production } \\
\text { Administrative } \\
\text { Ad hoc }\end{array}$ & Ad hoc & Production \\
\hline Model & $\begin{array}{l}\text { Tasks, Forms, } \\
\text { Roles, Users }\end{array}$ & $\begin{array}{c}\text { Job, Tasks, } \\
\text { Roles, Users }\end{array}$ & $\begin{array}{c}\text { Case, Procedure, } \\
\text { Roles, Users }\end{array}$ & $\begin{array}{l}\text { Map, } \\
\text { Submap, } \\
\text { Activity, } \\
\text { Couriers }\end{array}$ & $\begin{array}{l}\text { Tasks, } \\
\text { jobs, } \\
\text { Forms, } \\
\text { Roles, } \\
\text { Users } \\
\end{array}$ & $\begin{array}{l}\text { Map, Loop, } \\
\text { Act, Roles, } \\
\text { Users }\end{array}$ \\
\hline Client/Server based & YES & YES & YES & YES & YES & YES \\
\hline $\begin{array}{l}\text { Integration and lauch } \\
\text { of Office Applications }\end{array}$ & $\mathrm{NO}$ & YES & YES & YES & YES & $\begin{array}{c}\text { YES (via } \\
\text { Lotus Notes) }\end{array}$ \\
\hline Scripting Languages & $\begin{array}{l}\text { YES (php \& } \\
\text { JavaScript) }\end{array}$ & YES & YES & $\mathrm{NO}$ & $\mathrm{NO}$ & YES \\
\hline API Languages & $\mathrm{NO}$ & $\mathrm{C}, \mathrm{C}++$ & $\mathrm{NO}$ & $\mathrm{C}, \mathrm{XDP}, \mathrm{AD}$ 4GL & $\mathrm{C}, \mathrm{C}++$ & $\mathrm{C}, \mathrm{C}++$ \\
\hline Transport Mechanism & $\begin{array}{l}\text { RDBMS } \\
\text { (MySQL) }\end{array}$ & RDBMS & Email & RDBMS & \begin{tabular}{|c|} 
Proprietary \\
DB \\
\end{tabular} & Email \\
\hline $\begin{array}{l}\text { Forms Creation } \\
\text { Environment }\end{array}$ & YES & $\mathrm{NO}$ & YES & $\mathrm{NO}$ & YES & YES \\
\hline $\begin{array}{l}\text { Event Signaling } \\
\text { (Event-Action Triggers }\end{array}$ & YES & YES & YES & YES & YES & YES \\
\hline $\begin{array}{l}\text { Role/User } \\
\text { Administration }\end{array}$ & YES & YES & YES & YES & YES & YES \\
\hline Routing & $\begin{array}{c}\text { Conditional \& } \\
\text { Rule-based }\end{array}$ & $\begin{array}{c}\text { Conditional, } \\
\text { Parallel, } \\
\text { Rule-based } \\
\end{array}$ & $\begin{array}{c}\text { Conditional, } \\
\text { Parallel, } \\
\text { Rule-based } \\
\end{array}$ & $\begin{array}{c}\text { Conditional, } \\
\text { Parallel, } \\
\text { Rule-based } \\
\end{array}$ & \begin{tabular}{|c|} 
Conditional, \\
Parallel, \\
Rule-based \\
\end{tabular} & $\begin{array}{c}\text { Conditional, } \\
\text { Parallel, } \\
\text { Rule-based } \\
\end{array}$ \\
\hline $\begin{array}{l}\text { Graphical Workflow } \\
\text { Definition / } \\
\text { Manipulation } \\
\end{array}$ & $\mathrm{NO}$ & YES & YES & YES & YES & YES \\
\hline $\begin{array}{l}\text { Alarm/Deadline/ } \\
\text { Priority }\end{array}$ & YES & YES & YES & YES & YES & YES \\
\hline Process Tracking & YES & YES & YES & YES & YES & YES \\
\hline Concurrency Control & YES & YES & YES & $\mathrm{NO}$ & $\mathrm{NO}$ & YES \\
\hline Support for Recovery & $\mathrm{NO}$ & $\mathrm{NO}$ & $\mathrm{NO}$ & $\mathrm{NO}$ & $\mathrm{NO}$ & $\mathrm{NO}$ \\
\hline $\begin{array}{l}\text { Transaction } \\
\text { Coordination } \\
\end{array}$ & YES & $\mathrm{NO}$ & $\mathrm{NO}$ & $\mathrm{NO}$ & $\mathrm{NO}$ & $\mathrm{NO}$ \\
\hline Security & \begin{tabular}{|l|} 
Auditability, \\
Authentication, \\
Encryption \\
\&Users Rights/ \\
Privilege Control
\end{tabular} & Auditability & $\begin{array}{c}\text { Auditabity, } \\
\text { Electronic } \\
\text { signature, } \\
\text { Authentication }\end{array}$ & $\begin{array}{l}\text { Auditability, } \\
\text { Authenti- } \\
\text { cation }\end{array}$ & $\begin{array}{c}\text { Authenti- } \\
\text { cation, } \\
\text { Encryption. }\end{array}$ & \begin{tabular}{|} 
Auditability, \\
Authenti- \\
cation \& \\
Data \\
Access \\
Control \\
\end{tabular} \\
\hline $\begin{array}{l}\text { Reporting } \\
\text { Capabilities } \\
\end{array}$ & YES & YES & YES & YES & YES & YES \\
\hline $\begin{array}{l}\text { Keyword Search } \\
\text { and Workflow } \\
\text { Querying }\end{array}$ & $\mathrm{NO}$ & YES & YES & YES & YES & YES \\
\hline
\end{tabular}


Table 1 shows the features / product comparison between the developed automated workflow system and five commercial workflow management systems reviewed.

In product classification of workflow systems, our workflow system was designed and developed to perform strictly administrative task, while others can perform production, administrative and ad hoc task. Under model task, forms, roles and users are accepted by our application. All the workflow systems studied including our workflow system are client/server based application, that is, the client sends request to the server, and the server responds back. Our developed application used (php and JavaScript) scripting languages, this made our application very interaction and dynamic.

Under Application Programming Interface (API) languages, the proposed system did not make use of any API, because APIs add to the workload, overhead run time when used. All our codes were scripted using scripting languages like php and JavaScript which are light weight, as compared to others using $\mathrm{C}, \mathrm{C}++, \mathrm{AD} 4 \mathrm{GL}$ that are heavy weight. The proposed workflow system also supports event signaling by notifying users of a new event in queue as they login to that particular area. Effective routing of documents from one user to another, in order to specify how work will progress from one step to the next, you define routes between the steps in a workflow definition; our application performs this routing automatically using conditional and rule-based concept, while others uses parallel, conditional and rule-based routing concept. Our application has no keyword search because it was designed and developed to solve a narrow and particular purpose as compared to others that are general purpose. Our workflow system does not support graphical workflow definition; we combined the power of php and JavaScript in building and defining our workflow, while other supports graphical means like workflow specification language, and so on.

\section{Conclusion}

We have achieved our objective in designing the distributed environment automated workflow software architecture for loan transaction processing and also translating the design blueprint into real life software prototype using light weight web development tools. Test results reveal that the automated workflow system carefully eradicated the delay in the end-to-end processing of loan transactions. The automated workflow system also eradicated the computational errors associated with the traditional method. The automated workflow system was able to store, retrieve, and secure records more effectively and efficiently. Users were also able to apply for loan facilities from the comfort of their homes and track the status of their application online any time and from anywhere in the world.

\section{Acknowledgement}

The authors would also like to acknowledge the management and staff of following the welfare and loan schemes in Ambrose Alli University, Ekpoma, Edo State, Nigeria; ASUU Welfare and Loan Scheme, SSANU Welfare and Loan Scheme, NAAT Welfare and Loan Scheme, NASU Welfare and Loan Scheme, and finally, AAU Multipurpose Cooperative Society for sharing their data, insights, and time.

\section{References}

1. Aalst, M. P., Interorganizational Workflows: An Approach based on Message Sequence Charts and Petri Nets. Systems Analysis Modeling Simulation. 34(3), 335-367 (1999a).

2. Aalst, M. P., Inheritance of Interorganizational Workflows: How to Agree to Disagree Without Loosing Control? Information Technology and Management. 4, 345-389 (2003).

3. Aalst, M. P. and Weske, M., The P2P Approach to Inter-Organizational Workflows. In Proceedings of the 13th International Conference on Advanced Information Systems Engineering, CAiSE 2001, pages 140-156 (2001).

4. Aalst, M. P.; Lohmann, N.; Massuthe, P.; Stahl, C. and Wolf, K., Multiparty Contracts: Agreeing 
and Implementing Interorganizational Processes. The Computer Journal. 53(1), 90-106 (2010b).

5. Bhattacharya, K.; Caswell, N. S.; Kumaran, S.; Nigam, A.; and Wu, F. Y., Artifact-centered operational modeling: lessons from customer engagements. IBM Syst. J. 46, 703-721 (2007a).

6. Bravetti, M and Zavattaro, G., Contract Based Multi-party Service Composition. In International Symposium on Fundamentals of Software Engineering (FSEN). 4767, 207-222 (2007).

7. Das, S.; Kochut, K.; Miller, J.; Sheth, A.; and Worah, D., ORBWork: A Reliable Distributed CORBA-based Workflow Enactment System for METEOR2. Technical report, The University of Georgia (1996).

8. Fdhila, W.; Yildiz, U and Godart, C., A flexible approach for automatic process decentralization using dependency tables. International Conference on Web Services. 124.

9. Fu, X.; Bultan, T. and $\mathrm{Su}$, J., Realizability of Conversation Protocols With Message Contents. In Proceedings of the IEEE International Conference on Web Services, Washington, DC, USA. 96 (2004b).

10. Hofstede, A.; Glabbeek, R. V. and Stork, D., Query Nets: Interacting Workflow Modules That Ensure Global Termination. In Business Process Management. Springer Berlin, Heidelberg (2003).

11. John-Otumu, A. M., Okonigene, R. E., and Imhanlahimi, R. E., Architecture and Development of an Automated Workflow System for Employees' Savings \& Loan Scheme in Nigerian Universities, Quest Journal of Software Engineering and Simulation, 2(11): 6 - 16. Available at: www.questjournals.org/jses/papers/vol2issue11/B2110616.pdf(2015).

12. Khalaf, R., and Leymann, F., Role-based Decomposition of Business Processes using BPEL. In Web Services, International Conference. 770-780(2006).

13. Kindler, E.; Martens, A.; and Reisig, W., Interoperability of Workflow Applications:
Local Criteria for Global Soundness. In Business Process Management, Models, Techniques, and Empirical Studies, London, UK. 235-253 (2000).

14. Kowalski, R., Database updates in the event calculus. J. Log. Program. 12(1-2): 121-146 (1992).

15. Leymann, F. and Roller, D., Production Workflow - Concepts and Techniques. Prentice-Hall, Upper Saddle River, New Jersey (2000).

16. Liu, R.; Bhattacharya, K and Wu, F., Modeling Business Contexture and Behavior Using Business Artifacts. In John Krogstie, Andreas Opdahl and Guttorm Sindre, editors, Advanced Information Systems Engineering. 4495: 324-339 (2007).

17. Martens, A., Analyzing Web Service Based Business Processes. In Fundamental Approaches to Software Engineering. Springer Berlin (2005).

18. Mitra, S.; Kumar, R. and Basu, S., Optimum Decentralized Choreography for Web Services Composition. In Proceedings of the 2008 IEEE International Conference on Services Computing (2008).

19. Mohan, C.; Agrawal, D.; Alonso, G.; Abbadi, A. E.; Guenthoer, R. and Kamath, M., Exotica: $a$ project on advanced transaction management and workflow systems. SIGOIS Bull. 16, 45-50 (1995).

20. Nanda, M. G.; Chandra, S. and Sarkar, V., Decentralizing execution of composite web services. SIGPLAN Not. 39, 170-187 (2004).

21. Nigam, A. and Caswell, N. S., Business artifacts: An approach to operational specification. IBM Syst. J. 42, 428-445 (2003).

22. Paul, S.; Park, E. and Chaar, J., RainMan: a workflow system for the internet. In Proceedings of the USENIX Symposium on Internet Technologies and Systems on USENIX Symposium on Internet Technologies and Systems (1997).

23. Ranno, F. and Shrivastava, S. K., A Review of Distributed Workflow Management Systems. In Proceedings of the international joint conference on Work activities coordination and collaboration 
(1999).

24. Reichert, M. and Bauer, T., Supporting Ad-Hoc Changes in Distributed Workflow Management Systems. In Robert Meersman and Zahir Tari, editors, On the Move to Meaningful Internet Systems 2007: CoopIS, DOA, ODBASE, GADA, and IS. 4803, 150-168 (2007).

25. Reichert, M. U.; Bauer, T. and Dadam, P., Flexibility for Distributed Workflows. In Handbook of Research on Complex Dynamic Process Management: Techniques for Adaptability in Turbulent Environments. 137-171 (2009).

26. Rinderle, S.; Wombacher, A. and Reichert, M., Evolution of Process Choreographies in DYCHOR. In On the Move to Meaningful Internet Systems 2006: CoopIS, DOA, GADA, and ODBASE, 4275: 273-290(2006).
27. Shrivastava, W.; Wheater, S. M.; Shrivastava, S. K. and Ranno, F., A CORBA Compliant Transactional Workflow System for Internet Applications. In Proc. Of IFIP Intl. Conference on Distributed Systems Platforms and Open Distributed Processing, Middleware 98, SpringerVerlag. 1-85233 (1998).

28. Wodtke, D. and Weikum, G., A Formal Foundation for Distributed Workflow Execution Based on State Charts. In Proceedings of the 6th International Conference on Database Theory, London, UK. 230-246 (1997).

29. Yi, X. and Kochut, K. J., Process composition of web services with complex conversation protocols. In Design, Analysis, and Simulation of Distributed Systems Symposium at Adavanced Simulation Technology (2004a). 